\title{
CREENCIAS Y PRÁCTICAS POPULARES RELACIONADAS CON EL ORIGEN Y TRATAMIENTO DE LA LEUCEMIA
}

Ximena Palacios Espinosa *

\section{Resumen}

La mediación de las creencias en la conducta humana es una constante en el contexto de la consulta psicológica. Su identificación y abordaje terapéutico resultan fundamentales en el esfuerzo por procurarle al paciente mayores probabilidades de ajuste y adaptación durante el proceso de enfermedad. Esto es, un afrontamiento proactivo. En el presente documento se exponen algunas de las creencias y prácticas populares que realizan los pacientes con leucemia y que el autor ha identificado en su práctica clínica. Así mismo, se presentan en forma breve las razones por las cuales una persona genera y mantiene falsas creencias, se discute permanentemente sobre los efectos de estas en el afrontamiento de la enfermedad, para terminar con la presentación de una alternativa en la intervención psicológica de las creencias falsas en el contexto de la psicología de la salud.

Palabras clave: creencias y prácticas populares, leucemias, cáncer, psicología.

Abreviaturas: LLC, leucemia linfoide crónica.

\section{Presentación y análisis}

La mediación de las creencias en la conducta humana es una constante en el contexto de la consulta psicológica. Condiciones como la enfermedad actúan precipitando prácticas asociadas con las creencias de los pacientes y depende en gran medida del significado que le den a las mismas, el fortalecimiento y mantenimiento de ambas, lo que se traduce en el afrontamiento adaptativo o desadaptativo de la afección.

El cáncer ha sido una entidad históricamente estigmatizada que se ha asociado con muerte, dolor y sufrimiento. Suele considerarse como una "condena" de la que el individuo jamás tendrá posibilidad de escape. Por lo tanto el abordaje de los aspectos psicológicos, sociales y culturales relacionados con esta afección es de inmensa complejidad. También, de manera tradicional se han nutrido creencias,

Fecha recibido: mayo 27 de 2006

Fecha aceptado: junio 28 de 2006

Psicóloga. Mg.Psicología clínica y de la salud. Profesor auxiliar de carrera. Programa de Psicología, Facultad de Medicina Universidad del Rosario. actitudes y prácticas que intentan dar explicación al origen de la lesión e intentan encontrar opciones alternativas para su tratamiento.

De hecho, el lento progreso en el descubrimiento de la etiología, pero en especial las limitantes en el tratamiento que solo hace poco tiempo permiten el alcance de la remisión total, parecen ser las principales razones que mantienen el inagotable repertorio de creencias sociales.

Si bien este conjunto de enfermedades denominado cáncer ha sido en general estigmatizado, algunos de sus tipos lo han sido mucho más por el significado e impacto que generan sobre la persona. Es el caso del cáncer ginecológico (seno, ovario y útero en la mujer) y de los de testículo y pene en el hombre, porque confrontan a la persona con algunos aspectos asociados con su rol de género, el del cerebro por comprometer las competencias cognoscitivas y generar incapacidad para desempeñarse en forma cabal en comunidad y los tumores calificados como no sólidos o blandos tales como la leucemia. De hecho, el cáncer ha sido asociado con tumores sólidos que se ven o que se sienten y no con esta 
condición proliferativa que afecta la sangre, cuyo significado de por sí es tan complejo como el del mismo cáncer. Por lo anterior, las leucemias han sido objeto de elaboraciones sociales que influyen en su afrontamiento, dando lugar a la emisión de prácticas populares durante todo el curso de la enfermedad.

Las creencias en sí mismas están precedidas por los valores que no son otra cosa que principios amplios y generales que les sirven de base. Son proposiciones abstractas acerca de cómo deben ser las cosas, mientras que las creencias pueden definirse como enunciados específicos que las personas consideran ciertos. Es esta última característica de veracidad que debe discutirse en profundidad cuando de la funcionalidad de las creencias se trata. Lo cierto es que tanto los valores como las creencias influyen en la manera en que el ser humano ve e interpreta su entorno y, por lo tanto, forman la esencia de su personalidad; y como tal, tienen un valor, una trascendencia que debe ser siempre considerada, respetada y reconocida en cada persona. Podría decirse entonces que el hombre es en función de lo que cree $\mathrm{y}$ de lo que hace.

No obstante, es necesario interrogarse sobre la función adaptativa que tienen las creencias en el ser humano. Como cualquier elemento presente en la existencia humana, pueden ser adaptativas cuando favorecen actitudes positivas que promueven conductas funcionales y benéficas en la persona y su entorno. Es decir, que serán adaptativas en la medida en que promuevan comportamientos prosociales, decrementen el conflicto, favorezcan la asimilación y la acomodación y por lo tanto, el ajuste de una persona a un contexto, mientras no atenten contra su integridad física y mental, los costos de tenerlas no superen los beneficios y las prácticas que se deriven de éstas no comprometan la calidad de vida de quienes las mantienen.

Por consiguiente, serán desadaptativas en cuanto vayan en dirección opuesta a lo antes mencionado, lo que implica que vayan en detrimento del bienestar físico y mental de quien las posea, dificulten o interfieran en la toma de decisiones funcionales, en tanto los costos superen los beneficios de las prácticas que se derivan de ellas, en tanto dificulten el ajuste y la adaptación de un ser humano a las situaciones difíciles y estresantes o interfieran en forma negativa en la percepción de los eventos vitales.

Como se mencionó, la creencia es un enunciado específico que una persona considera cierto, cuyo concepto de veracidad debe ser analizado. Precisamente porque a partir de una creencia es que el hombre forma una serie de impresiones que por ende, considera ciertas, verdaderas. Y como tal, hará interpretaciones basado en ellas. Lo anterior no tendría lugar a discusión si no se estuviera frente al hecho de que la creencia en sí misma y per se, es creación del humano y que está basada en el sentido común. Es el resultado del procesamiento de la información que hace el hombre a partir de su experiencia y que se confirma también a través de la experiencia. Una creencia suele carecer de una base sólida de conocimiento y se retroalimenta de la experiencia social del ser humano.

De acuerdo con Myers (1996), las creencias son sistemas socializados de conceptos e ideas, que organizan la percepción de partes del mundo (o de su totalidad) en el que vive la sociedad de referencia. Pueden contener componentes míticos (cifrados sobretodo en las relaciones de parentesco utilizadas para enlazar los fenómenos cósmicos) o religiosos, pero también hay creencias no míticas sino "racionalizadas" (por ejemplo, la creencia en la esfericidad del mundo físico) sin que por ello sean verdaderas.

De acuerdo con Moscovici (1973) citado por León (1998), las creencias o representaciones sociales son sistemas cognoscitivos con una lógica y lenguaje propios. No significan solo opiniones, imágenes o actitudes en relación con algún objeto, sino teorías o áreas de conocimiento para el descubrimiento y organización de la realidad. Este sistema de valores, ideas y prácticas asume dos funciones: 1) establecer un orden que permita a los individuos orientarse en su mundo material y social para dominarlo y controlarlo; y 2) permitir la comunicación entre los miembros de su comunidad al proveerlos de un códi- 
go para el intercambio social, con el fin de nombrar y clasificar aspectos de su mundo y de su historia personal y grupal. Pero además de ejercer funciones en el área cognoscitiva, también pueden influenciar las dimensiones de naturaleza afectiva, como clasificar, explicar y evaluar los objetos sociales, creando entonces una orientación para la acción. De acuerdo con esto, las representaciones sociales parecen actuar como un metasistema de regulaciones sociales, que intervienen en el funcionamiento del sistema cognoscitivo y afectivo e influyen en la organización del sistema de creencias y conocimientos de aspectos de la realidad para un grupo (Doise, 1993, citado por León, 1998).

Es decir, que la noción de representación social es amplia, de carácter integrado, y se presenta bajo formas variadas, más o menos complejas. Imágenes que condensan un conjunto de significados; sistemas de referencia que permiten interpretar lo que sucede, e incluso dar un sentido a lo inesperado; categorías que sirven para clasificar las circunstancias, los fenómenos y los individuos; teorías que permiten establecer hechos entre ellos (Gutiérrez, 1998).

A partir de las creencias, la persona se forma impresiones y hace interpretaciones de la realidad; pero si éstas son falsas, seguramente también lo serán la impresión y la interpretación que haga, por lo que pueden generarse, entre otras consecuencias, dudas, confusión, temores, conflicto personal e interpersonal, inconformidad y por consiguiente, van en detrimento de la salud física y mental. Uno de los interrogantes más importantes en relación con las falsas creencias es el de cómo se forman y se mantienen. De acuerdo con Myers (1996), hay seis respuestas al respecto, las cuales se expondrán a continuación.

1. Con frecuencia los seres humanos actúan sin saber por qué lo hacen, a lo que por lo general subyace la necesidad de hallar posibles respuestas a aquello que le está generando conflicto. Así, cuando esto último suele no tener un origen, una causalidad ni unos determinantes claros, las explicaciones que la persona se da a sí misma suelen ser contradictorias y en consecuencia pueden ignorarse o considerarse inocuos aquellos factores que tienen grandes efectos sobre la explicación del problema y por el contrario, se consideran muy influyentes aquellos factores que tienen poco o ningún efecto sobre el problema. Es el caso de los pacientes oncológicos que deciden abandonar una sesión de quimioterapia, o muchas veces incluso el tratamiento definitivamente, tras la experiencia de la acatisia provocada por antieméticos de tipo metoclopramida. Esta reacción adversa bien conocida, suele ser poco advertida a los pacientes, quienes en el caso del contexto oncológico, la experimentan con significativa frecuencia. Por consiguiente, ante el particular malestar experimentado en forma de inquietud intensa, desesperación, deseo y necesidad de escapar y sensación de pérdida de control, el paciente tiene, como es de esperarse, una respuesta de escape y evitación que le proporciona refuerzo negativo por la retirada de ese estímulo aversivo y que se consolida como un factor de mantenimiento de la conducta.

Lo anterior se traduce en el establecimiento o confirmación de una falsa creencia, muy común desde que el cáncer se ha estigmatizado: "la quimioterapia es algo terrible que produce gran sufrimiento en quien la recibe". Ya que no se actúa desde la prevención primaria advirtiendo al paciente de este posible efecto medicamentoso, la acción terapéutica se hace desde el contexto de la prevención terciaria.

Por consiguiente, cuando el paciente ya ha tenido la experiencia aversiva y han tenido lugar las respectivas consecuencias, suele explicársele que esto fue una reacción adversa del medicamento antiemético (lo que el paciente considera como un factor inocuo) y que su malestar y sufrimiento (factor influyente) no volverán a tener lugar con el cambio o diferente administración del medicamento. Así que el resultado final se traduce en el abandono o aplazamiento del tratamiento o en su defecto, en la negativa influencia sobre el compromiso (adherencia) con el mismo.

Por lo tanto, es probable que tras un paciente con ausencia de compromiso con el tratamiento, haya una o varias experiencias negativas que expliquen la 
conducta pero que el paciente en sí mismo no sabe lo que hace si al final, su objetivo y su deseo, al igual que el del médico tratante, también es curarse. O que pueda explicar la razón por la que abandonó el tratamiento convencido de que fue la peor experiencia de su vida y de que su justificación es válida y su falsa creencia confirmada o adquirida de que: "la quimioterapia es algo terrible que produce gran sufrimiento en quien la recibe", es suficiente para abandonar o no comprometerse cabalmente con su tratamiento. En conclusión, con gran facilidad el ser humano puede explicar en forma falsa la razón o razones de su conducta.

2. La segunda explicación al interrogante de la formación y el sostenimiento de las falsas creencias está en el enorme efecto de las preconcepciones sobre las interpretaciones y recuerdos de las cosas. Al respecto hay que anotar que el ser humano tiene una particular capacidad mnésica sobre otras especies y su capacidad de juicio admite la de preconcebir. De hecho, siempre que una persona intenta entender o dar significado a algo, lo hace a partir de una idea o de un conocimiento adquirido antes. Por lo tanto, la influencia de las expectativas e ideas alternativas sobre la forma en que una persona actúa y percibe el mundo, es un rasgo característico del funcionamiento cognoscitivo del hombre. Casi todo lo que hacen las personas está determinado por la manera en que intentan darle sentido a las cosas que les suceden y a las personas que las rodean. Sin embargo, sobre una misma realidad pueden darse distintas descripciones de acuerdo con las necesidades funcionales de la organización que cada persona hace de su mundo. Estas necesidades por lo general prefieren las explicaciones causales $\mathrm{y}$ es porque de esta manera el ser humano puede predecir y controlar los acontecimientos vitales, y estas dos acciones son adaptativas.

3. Otra de las razones por las cuales se mantienen las falsas creencias se explica por la sobreestimación que el individuo hace de la precisión de sus juicios. Esa confianza excesiva puede traducirse en consecuencias que van en detrimento de la salud física y mental del paciente, que se siente más confiado que acertado y dirige sus esfuerzos a buscar información que confirme su creencia.

Es el caso de una paciente, mujer de cerca de 50 años de edad, que detecta una masa en su seno izquierdo que generaba lesiones cutáneas causantes de significativa incomodidad y que comenzaron a expedir un olor desagradable que la preocupaba mucho más que la propia masa, de la cual confió en que era un quiste. Decide buscar ayuda consultando a un practicante de la bioenergética, a quien antes había acudido con éxito y en quien confiaba plenamente y le indicó que la cura estaba en la utilización de emplastos de "barro de origen volcánico". En efecto, las lesiones desaparecieron y la paciente confirmó la eficiencia de las terapias bioenergéticas. No obstante, la masa no desapareció y al respecto su terapeuta asintió sobre la posibilidad de que fuera un quiste y que desaparecería también con el mencionado remedio. Cerca de un año después comienza a experimentar dolor lumbosacro de intensa magnitud (9/10) que impide la marcha y dificulta conciliar el sueño, por lo que es llevada a urgencias en donde las placas demuestran lesiones sugestivas de metástasis óseas. Hacen interconsulta a oncología que confirma el diagnóstico de cáncer de mama en estadio IV con metástasis pulmonares y óseas.

Por lo tanto, entre los objetivos psicoterapéuticos en relación con las creencias que definen confianza excesiva en los pacientes está reestructurar una de las principales ideas que subyacen a esta y es que a pesar de que una persona parezca segura de que tiene la razón, puede estar equivocada. Luego, se aboga por el beneficio de la duda. Entrenar al paciente para que aprenda a considerar qué razones pudieran estar indicando creencias equivocadas y buscar evidencia científica en lo posible de las razones por las que sus ideas o creencias son verdaderas.

4. Utilizar la información inútil e ignorar la útil es la cuarta razón por la que se forman y mantienen las falsas creencias. Por lo general, las situaciones de enfermedad pueden generar estados de confusión y desesperanza que hacen vulnerable al individuo ante el juicio que hace de los eventos cotidianos y por 
supuesto aún más, los asociados con la enfermedad. Por consiguiente, es fundamental advertir sobre la precaución que debe tenerse al juzgar la probabilidad de las cosas por la representación previa que se tenga de ellas.

De hecho, suele recordarse más la información inútil por el impacto que tiene sobre el individuo, lo que por lo general influye en forma negativa sobre la toma de decisiones. Esta es una condición frecuente en los pacientes oncológicos y en general en los crónicos durante el proceso de la enfermedad. Un ejemplo de ello se relaciona con la necesidad de saber qué pudo causar el cáncer. Una paciente con una neoplasia laríngea aseveraba que apareció después de haber comido un dulce de mora en el que había una astilla que se clavó en su garganta formando el tumor. Esta mujer, con historia de consumo de cigarrillo (una cajetilla diaria) durante más de 25 años, le dió un valor causal muy bajo, casi desapercibido, a la influencia del cigarrillo en la etiología del cáncer (información útil) y un alto valor a la astilla en el dulce de mora (información inútil). La decisión personal fue restringir por completo este tipo de alimento de su dieta, mientras que el cigarrillo lo abandonó por indicación médica y presión familiar.

Decidir no hacer el tratamiento oncológico ignorando sus efectos antitumorales (información útil) por el temor generado a partir de lo que los vecinos le han comentado acerca de los efectos colaterales que puedan experimentarse (información inútil), es otro ejemplo que ilustra esta cuarta razón para mantener falsas creencias.

5. Es posible que uno de los comportamientos con mayor dificultad en lo que a su modificación concierne, es el que está relacionado con la superstición. Los seres humanos son la única especie con esa necesidad de buscarle un orden a los acontecimientos casuales. El objetivo es claro: poder predecir y controlar las situaciones cotidianas. Por consiguiente, si la persona cree que las premoniciones se relacionan con los eventos, observará y recordará la relación de la premoción y la ocurrencia posterior a este evento, y tenderá a omitir o a atenuar la evidencia de todas aquellas veces en las que los eventos inusuales no coinciden. Sin embargo, la necesidad que tiene el ser humano de predecir y de controlar, suele dar lugar a aquello que se conoce como la ilusión de control. Myers (1995) define este concepto como la percepción de eventos incontrolables que tratan de estar sujetos al control de la persona o como más controlables de lo que en realidad son. Independiente del nivel educativo, la cultura, la raza o cualquier otra cantidad de variables, la ilusión de control puede desarrollarse y mantenerse en las personas. Al respecto es necesario anotar que la explicación precede al control y para explicar es fundamental conocer las causas de los acontecimientos. Por lo tanto, las situaciones vitales adquieren significado de acuerdo con la experiencia de la persona que las experimenta y se fortalece de acuerdo con el aprendizaje y la historia personal del individuo. Lo anterior implica entonces que ideas preconcebidas tienen funciones adaptativas para la supervivencia del ser humano: 1) permiten predecir acontecimientos futuros tanto si son deseados como si son temidos y 2) permiten explicar o atribuir un efecto a una determinada causa. Así que lo grave en realidad no es enfermar sino la interpretación que se hace de la afección. Una opción es que a partir del diagnóstico la persona "sea" enferma y otra que "esté" enferma. Las consecuencias de estas dos variables son diferentes si la persona asume el rol de enferma o si considera que está enferma. La primera se constituiría prácticamente en un rasgo, mientras que la segunda implica temporalidad; es un estado.

Si la persona considera que las causas de la enfermedad no son controlables, se percibirá indefensa ante sus consecuencias. Por lo anterior es que resulta adaptativo en apariencia que las personas tengan la creencia de que pueden controlar aquello que está fuera de su control, es decir que tengan una ilusión de control. De hecho, el aparente control se ha relacionado en forma muy estrecha con el sufrimiento (Bayés, 2001), por lo que a mayor percepción de control, los eventos se interpretan como menos amenazantes y por lo tanto, la persona considera sufrir menos. Lo anterior explica que los pacientes establezcan rituales asociados al azar con el objeto 
de conseguir controlar los eventos relacionados con los temores hacia la enfermedad. Por ejemplo, una persona cree que puede controlar la recaída de una leucemia. Al respecto hay que anotar que se trata de una condición azarosa en la medida en que no se puede afirmar con un $100 \%$ de seguridad cuándo tendrá lugar una recaída o siquiera, si la tendrá. Aunque los estudios pueden demostrar el comportamiento epidemiológico de ello, solamente el curso natural y estrictamente biológico de la enfermedad, tendrán la respuesta a la aparición o no de una recaída. Algunos pacientes creen poder controlarla e incluso incurren en conductas de riesgo y se exponen a ellas porque tienen la falsa percepción de que están bajo su control y por ello, los libera de la vulnerabilidad biológica que subyace al tema de las recaídas.

6. La última razón por la que se mantienen las falsas creencias es que éstas pueden generar su propia realidad. Y ello da lugar a lo que se ha denominado la profecía autocumplida, es decir la tendencia a que las expectativas propias evoquen una conducta que las confirme (Myers, 1995). Así, cuando un paciente tiene bajas expectativas en relación con la terapia oncológica, posiblemente termine confirmando su pobre efectividad y a confirmar la respuesta del método bioenergético. Suponga que un paciente ha decidido asumir ambos tipos de tratamiento de forma simultánea pero ha desconfiado siempre del oncológico tradicional por los efectos colaterales experimentados. De hecho, percibe que la terapia alternativa le provee de bienestar y le alivia las molestias experimentadas con la quimioterapia. Ha tenido que hacer, tal y como lo supone el protocolo oncológico, una serie de exámenes de rutina para constatar el estado de activación tumoral y cuando llega a consulta con su oncólogo, éste le indica que hay presencia de metástasis, lo que confirma sus dudas acerca de la quimioterapia (profecía autocumplida); pero además, continúa con la medicina bioenergética que no solo le ofrece menores molestias de carácter fisiológico, sino que alivia potencialmente la percepción del sufrimiento experimentado tras la noticia de la progresión de su enfermedad. Por todo lo anterior, se puede predecir que el compromiso (adherencia) del paciente se dirigirá al tratamiento alternativo antes que al tradicional aunque ninguno de los dos prometa curación y se confirmará su expectativa de que el oncológico no solo es aversivo sino inútil y que el alternativo es deseable y necesario pues lo cierto es que la ciencia en la terapia del cáncer está aún limitada y por lo tanto, el oncólogo no puede prometer aquello que desconoce.

De las creencias que una persona tenga dependerán en gran medida sus actitudes (sentimientos de agrado o de desagrado que experimenta con respecto a la conducta) y de éstas, sus intenciones y su conducta. Así lo plantean diferentes modelos de la psicología de la salud como la teoría de la acción razonada (Fishbein y Ajzen, 1975). Este tipo de modelos constituyen una estructura conceptual unificada y sistemática que permite explicar la conducta humana. De hecho, los seres humanos (como animales superiores) tienen la capacidad de procesar y utilizar en forma permanente la información (creencias y actitudes) de una manera sistemática. Así, juzga, evalúa y toma decisiones.

Por consiguiente, las creencias se constituirán en el sustrato que da origen a las actitudes, mientras que las intenciones tienen como base dos determinantes: el personal y el social. Una conducta se realiza o no según la intención que a su vez está fundamentada en la actitud y en la norma y estas dos en las creencias conductuales y normativas. Pero lo interesante está en que cuando el ser humano realiza algunas conductas, éstas por sí mismas generan nuevas creencias y por lo tanto, se establece un ciclo de retroalimentación permanente.

Ante lo anterior habría entonces que mencionar que la traducción de que una actitud encuentre su etiología en las creencias, está en la convicción de la persona en relación con las consecuencias (positivas, negativas, adaptativas, desadaptativas, favorables, desfavorables, agradables, desagradables) que obtendrá a partir de la emisión de las conductas.

No obstante, y de acuerdo con Fishbein y Ajzen (1975) esto adquiere mayor complejidad en cuanto debe considerarse la subjetividad de las normas (per- 
cepción acerca de las presiones sociales impuestas en relación con el objeto) que la persona tiene hacia una conducta o en contra de la misma.

Así, tanto la personalidad como la expectativa que la persona tiene acerca de los refuerzos, gratificaciones o recompensas de su actuar (locus de control), sus creencias, actitudes e intenciones, definirán su estilo y estrategias de afrontamiento. Es decir, los recursos de los que disponga para resolver las situaciones que se le presenten en la cotidianidad, independiente de la importancia o significado que puedan tener objetiva o subjetivamente.

En lo que respecta a las creencias relacionadas con la leucemia, habría que anotar que estas suelen estar dirigidas a la etiología, al tratamiento y al pronóstico. Con respecto a la primera, el paciente suele preguntarse e intenta responder a ¿qué pudo haber causado esta enfermedad? Como producto del intercambio psicoterapéutico con el paciente oncológico y en forma específica con leucemia, se han identificado varias creencias falsas asociadas con la etiología (Tabla 1) que desencadenan prácticas populares que suponen algún tipo de riesgo para ellos y que por lo general están dirigidas a buscar la curación. Se hace énfasis en la curación física, porque otras son las prácticas populares que tienen un impacto sobre la salud mental de los pacientes, sobre el bienestar psicológico y la subjetividad de la interpretación de los eventos relacionados con la enfermedad, temas que no son el objeto de este artículo. Lo anterior no implica que las prácticas asociadas con la curación física no tengan impacto sobre la salud mental. Sin embargo, estas costumbres populares suelen poner en riesgo la vida y se relacionarán en alguna medida con su pronóstico.

Los pacientes con leucemia y sus familiares suelen insistir en que ésta se desarrolla en las personas con antecedentes de anemia. Al respecto, los únicos dos tipos de anemia que se han relacionado con las leucemias secundarias son la constitucional de Fanconi y la sideroblástica.
Creencias como que la leucemia está relacionada con antecedentes de metrorragia, que aparece en las personas que han donado sangre o en aquellas que han sido transfundidas, no tienen ningún referente de tipo científico que permita validarlas. No se encuentra evidencia de ninguna relación entre estas condiciones y el desarrollo de la leucemia. Lo único posible es que los leucémicos presenten hemorragias y las mujeres cursen con metrorragias. Pero como está claro, es una condición secundaria a la leucemia y no la antecede ni la origina.

La psiconeurinmunoendocrinología es una rama de la psicología que se encarga del estudio de las relaciones entre el comportamiento y la enfermedad; por lo tanto, intenta explicar la forma en que la conducta de una persona incide sobre los estados de salud-enfermedad que son modulados por el sistema nervioso, el sistema inmune y la función metabólica.

Dos de los principales temas de interés de ésta han sido el estrés y la depresión y sus efectos sobre la salud física de las personas. Si bien algunos estudios (Kietcolt-Glaser y Glaser, 1995; Witfield, 2002; kietcolt-Glaser et al., 2002; Raison, 2002; Miller et al., 2004; Lusk y Lash, 2005; Uhlig y Kallus, 2005; Fette, 2005; Bosch et al., 2005; Tosevski y Milovancevic, 2006; Vergara-Rodríguez, 2006) han demostrado que existe relación entre la inmunosupresión y el estrés, y sobre inmunosupresión y depresión (Lesperance et al., 2004; Bower et al., 2005; Motivala et al., 2005;) esta no es causal. Sucede igual con el cáncer (Kietcolt-Glaser, 1999; Savard et al., 1999; Prieto et al., 2002; Capuron, Gumnick y Miller, 2002; Tacon, 2003; Holland, 2003; Carlson et al., 2003; Pereira et al., 2003). Sin embargo, debe considerarse que el estrés puede interferir con estados óptimos de inmunocompetencia.

Por lo anterior, las creencias de que la leucemia se desarrolla a causa de eventos vitales estresantes (pérdida del trabajo, muerte, jubilación, crisis económicas, entre otros) o depresión, son falsas. E1 hecho de que experimente estados de estrés o de depresión durante la enfermedad no va a tener necesariamente 
consecuencias dramáticas sobre la evolución física del paciente, pero sí puede aumentar la probabilidad de que ésta no sea óptima. Lo mismo sucede con el sufrimiento. Si bien las personas que perciben altos niveles de sufrimiento pueden experimentar estados de estrés, ansiedad y depresión clínicamente significativos, bien como causa o consecuencia de éste, no hay ninguna razón para pensar en que sus efectos sobre el sistema inmune o el metabolismo de una persona fueran diferentes de aquellos que acaban de exponerse antes.

También sucede con los pensamientos negativos que pueda tener. Es posible que sean responsables del malestar psicológico (distrés) que ésta experimente, pero no los responsables del origen de una leucemia ni de ningún otro tipo de cáncer. De hecho, la evidencia solo menciona que el pensamiento puede actuar como variable moderadora dentro del proceso de enfermedad, haciendo que la persona afronte con recursos más o menos efectivos las situaciones vitales estresantes, entre ellas la enfermedad. Pero no existe ninguna comprobación científica de su directa relación con la etiología de la misma.

Con respecto a la creencia de que la leucemia es causada por la exposición a sustancias tóxicas, se sabe que existe una relación estrecha con el benceno* (industria del caucho, imprentas, petroquímica) (Milanés et al., 2002). En la actualidad, existen 22 productos químicos, grupos de sustancias o mezclas en las que la exposición es casi siempre profesional (excluidos los pesticidas y los fármacos), que son cancerígenos establecidos para el ser humano**. Evaluados en las monografías del IARC, volúmenes 1-63 (1972-1995) (excluidos pesticidas y fármacos). Algunos de estos compuestos (por ejemplo el amianto, el benceno y los metales pesados) son utilizados en muchos países del mundo. Ejemplo de ello son los insecticidas organoclorados como el DDT, el heptacloro y el lintano cuya relación con la leucemia se evidenció en los estudios de Holan y Spurling (1974), Nunes y Tajara (1998) y Torres y Capote (2004); también sucede con los insecticidas organofosfatados como el palatión y el malatión, ciertos herbicidas e incluso compuestos antineoplá- sicos como la azotioprina, busulfán, clorambucil, ciclofosfamida o melfalán que han sido asociados con el desarrollo de algunas leucemias. Otros como la procarbacina, mecloretamina, actinomicina, doxorrubicina, cisplatino, dacarbacina y nitrosoúreas lo han sido en menor medida. El consumo de algunos medicamentos también ha demostrado estar asociado con el desarrollo de leucemias. Es el caso del cloranfenicol (Mappilacherry y Chandra, 1990; Jiménez et al., 1990; Abbas, Malik y Kan, 1993; Doody et al., 1996; ), la fenilbutazona (Symmons, 1995; Duffy et al., 1998) y la oxifenbutazona.

Con respecto a si la leucemia se desarrolla por el uso del celular, los estudios como el de Hardel y Carlberg (2004) demostraron que es posible que haya un incremento del riesgo para desarrollar tumores cerebrales con el uso de teléfonos celulares análogos. Otros como el de Derias et al. (2006) no permiten establecer conclusiones sobre esta relación pero indican que es necesario continuar estudiando esta posibilidad, situación que se había planteado antes en los estudios de Frey (2002) y de Moulder et al. (2005).

En lo que respecta a las creencias religiosas relacionadas con el origen de la enfermedad, estas no son el objeto de este artículo, por lo que no se entrará en su discusión; no obstante, es necesario que se considere su trascendencia sobre el estado psicológico del paciente.

Por último, en lo que se refiere a la creencia de que la leucemia es el producto de un hechizo (brujería), no existe evidencia científica alguna que permita constatar la validez de una creencia que por sí misma está soportada en un imaginario colectivo que no encuentra explicaciones en la ciencia, por lo cual el profesional debiera declararse impedido para emitir juicios que no serían de corte profesional sino que pasarían a ser solo de carácter valorativo. Lo cierto es que la leucemia es una enfermedad, un estado de descompensación en la funcionalidad de la producción natural de elementos a partir de la médula ósea y que por consiguiente la explicación de su origen redunda en alteraciones de tipo biológico. Si se parte 
de este supuesto de enfermedad, se está trabajando en centrar la atención en las acciones dirigidas a resolver, o por lo menos a aliviar, ese desequilibrio y no en las causas de su origen.

\section{Tabla I. Falsas creencias relacionadas con la etiología de la leucemia.}

- Se desarrolla en las personas con antecedentes de anemia.

- Está relacionada con antecedentes de metrorragia.

- Aparece en las personas que han donado sangre.

- Surge en quienes han sido transfundidos.

- Se desarrolla a causa de eventos vitales estresantes (pérdida del trabajo, muerte, jubilación, crisis económicas, entre otros).

- Es causada por estados de estrés o de depresión.

- Se origina por la exposición a sustancias tóxicas.

- Se desarrolla por el uso del celular.

- Aparece por el sufrimiento que experimenta una persona.

- Se produce por tener pensamientos negativos.

- Es el producto de un castigo divino por malas acciones de la persona.

- Es el producto de un hechizo (brujería).

Nota.Tomado de la práctica clínica de la autora con el paciente con leucemia.

Algunos tipos como la leucemia linfoide crónica (LLC) son de origen desconocido (Hernández, 1999). Este mismo autor menciona que es de hecho la única leucemia del adulto en la que no se ha encontrado relación entre factores de exposición, radiaciones ionizantes, agentes químicos o virus, y su aparición. A favor de este criterio está el hecho de que no existen evidencias de que los japoneses supervivientes a las radiaciones producidas por la bomba atómica lanzada en su país hayan contraído un mayor riesgo de padecer LLC. Así mismo, los estudios realizados en gemelos univitelinos con LLC sugieren la naturaleza adquirida de la enfermedad (Montserrat, 1992).

También algunas condiciones congénitas se han vinculado al desarrollo de leucemias agudas: 1) el sindrome de Down (Crombet Ramos y Svarch Guerchicoff, 1998; NCI, 2005); 2) el sindrome de Bloom (Hojo et al., 1995; Gibbons et al., 1995; German, 1995; Bhisitkul y Rizen, 2004; Kaneko y Kondo, 2004); 3) la anemia constitucional de Fanconi (Gosdazoglu et al., 1980; Gibbons et al., 1995; Hojo et al., 1995); 4) la ataxia - telangiectasia (Hojo et al., 1995); 5) la trisomia D o Sindrome de Paton; 6) el sindrome de Wiscott-Aldrich (Atra et al., 1998; Mateo et al., 2002) y 7) la agammaglobulinemia (Hensel et al., 2003; Newcom, 2005).

La evolución de algunas hemopatías puede favorecer el desarrollo de leucemias agudas. Tal es el caso de: 1) la policitemia vera; 2) la leucemia mieloide crónica; 3) la mielofibrosis; 4) la anemia sideroblástica (Padua y West, 2000; Maghalaes et al., 2000; D'Angelo, 2005); 5) la hemoglobinuria paroxística; 6) el mieloma múltiple (Pasqualetti et al., 1997; Eclache et al., 1993; Krosravi, 2005); 7) la LLC y 8) la enfermedad de Hodgkin.

Las creencias acerca de que la leucemia se desarrolla en personas anémicas es en parte cierta. Así se ve en la anemia constitucional de Fanconi y la sideroblástica. Las anemias de otro tipo, no. Por lo demás, estas dos variedades son poco comunès en la población general.

Parece existir una etiología asociada con dos tipos específicos de virus en las leucemias agudas: 1) el de la mononucleosis infecciosa que está relacionado con el linfoma de Burkitt y con la leucemia aguda tipo Burkitt, y 2) el virus linfotropo humano (HLTVI) relacionado con la leucemia aguda por linfocitos T (Borducci et al., 1998; Kishi et al., 2001; Barros, 2006).

Como es conocido, las radiaciones ionizantes están muy relacionadas con el desarrollo no solo de la leucemia sino de otros tipos de cáncer (Auvinen et al., 1994; Rahu et al., 1997; McNally y Parker, 2006; Wrigth y Coates, 2006; Rahu et al., 2006; Gluzman et al., 2006). Sin embargo, una evidencia importante de la relación entre éstas y la leucemia, está en el comportamiento epidemiológico que presentara esta enfermedad en la población de Hiroshima y Nagasaki tras la explosión de las bombas atómicas (Breckow, 
1991; Helmberg, 1992; Delongchamp et al., 1997; Preston et al., 2004; Muirhead, 2003), donde se vió un aumento muy significativo de su incidencia hasta hace pocos años.

Es posible que el mayor conocimiento del origen radica en la leucemia mieloide crónica, de la cual se conoce que su etiología está relacionada con la presencia del cromosoma Filadelfia (Ph1) (Schwarz et al., 2006; Valle te al., 2006; Campbell, 2006).

Lo anterior permite concluir que hay algunos factores de riesgo identificados para explicar el origen de las leucemias. No debe olvidarse el componente genético ni tampoco que estos factores no tienen un efecto causal directo sino que posiblemente aumenten la vulnerabilidad para el desarrollo de esta enfermedad.

En lo que respecta al tratamiento de la leucemia, existe una serie de creencias populares que se transmiten promoviendo la presentación y establecimiento de prácticas dirigidas a la curación o el alivio de la sintomatología propia de la enfermedad (Tabla 2).

El desarrollo de este tipo de prácticas tiene un sustrato cultural de inmensa importancia que no solo es necesario conocer sino comprender. Es tarea del psicólogo y en general de todos los miembros del equipo de salud, destinar un tiempo prudencial para analizar las razones que están determinando la necesidad de un paciente al realizarlas. No obstante, si bien es respetable su desarrollo, el profesional no puede ignorar que su función y deber con el paciente está en la orientación hacia un proceso de toma de decisiones funcionales y adaptativas. Por lo tanto, la conclusión que puede extraerse de esta relación entre las creencias y las prácticas populares, es que son inherentes al ser humano pero así mismo, pueden ser funcionales o disfuncionales y como tal, desencadenar en acciones que vayan en detrimento de la salud física y emocional del paciente.

Hasta el momento, la terapia hemato-oncológica para el manejo de la leucemia está fundamentalmente centrada en la quimioterapia. Es el tratamiento de elección y encuentra soporte coadyuvante en la radioterapia y en ocasiones en los procedimientos quirúrgicos en caso de requerirse esplenectomía. Sin embargo, también han surgido una serie de terapias denominadas alternativas que deben ser comprendidas como complementarias y no con capacidad de reemplazo de la oncológica tradicional.

Contrario a lo que sucede con la terapia tradicional, la alternativa suele generar altos niveles de compromiso (adherencia) por parte del paciente. Hay varias explicaciones al respecto, pero desde el contexto que compete a las creencias y el afrontamiento, habría que partir de que la creencia que subyace al tema de la terapia alternativa es que se trata de una opción diferente, que dista de causar los efectos colaterales de la quimioterapia. Los costos en relación con los beneficios son mucho menores. Es cierto que nadie va a vomitar con la primera como con la segunda y que es posible que no cause ni alopecia ni inmunosupresión. Por supuesto, la percepción de sufrimiento será menor cuando le ofrecen una alternativa de carácter menos invasivo, pero quizás también menos efectivo. Ante enfermedades de estas características tan inciertas en lo que a su etiología y pronóstico supone, las promesas son mayores que las certezas y esto da lugar a la asunción de opciones que implican menos riesgo psicológico.

El Instituto Nacional del Cáncer (2005) ha aceptado algunas prácticas como parte de las terapias alternativas pues ha reconocido el efecto potencialmente benéfico que tienen sobre el paciente. Entre estas se encuentran la quiropraxia, la relajación, la meditación y la acupuntura. Al respecto, el Instituto Nacional del Cáncer y el Centro Nacional para la Medicina Complementaria y Alternativa patrocinan o co-patrocinan varios estudios clínicos para estudiar los tratamientos complementarios y alternativos para cáncer.

Algunos de estos estudios tratan de los efectos de los métodos complementarios que se usan además de los tratamientos convencionales, mientras que otros comparan las terapias alternativas con las convencionales. Los estudios actuales son los siguientes (NCI, 
Tabla 2. Falsas creencias relacionadas con el tratamiento de la leucemia y algunas de las prácticas derivadas de estas

\section{Creencia}

- La quimioterapia es veneno.

- La quimioterapia es peor que la enfermedad.

- La quimioterapia no sirve, es experimentación.

- El hierro cura la leucemia pues esta es producto de la anemia.

- La sangre es el elemento que se requiere para la curación de la leucemia.

- La brujería puede combatir la enfermedad pues esta es producto de hechizos.

- La leucemia puede ser curada por fuerzas sobrenaturales.

- Los productos naturales tienen el poder de curar la leucemia.
- Respuesta/ práctica popular

- Rechazar la quimioterapia y acceder a tratamientos alternativos.

-Tomar jugo de mora con hígado crudo licuado

- Comer morcillas.

- Comer vísceras (hígado, pulmones, intestinos, corazón), tuétano de los huesos y cabezas de pescado; por lo general preparadas en caldo o cocidas.

- Tomar sangre de animales; específicamente hay una práctica muy común relacionada con el consumo de sangre de chulo.*

- Consultar a rezanderos y hacer rituales dirigidos a liberar a la persona del efecto del hechizo realizado.

- Prácticas de índole religioso, en especial en relación con las intervenciones de José Gregorio Hernández.**

Ingerir extractos de plantas o de sus frutos como el anamú o el noni.

Nota.Tomado de la práctica clínica de la autora con el paciente con leucemia.

2005): 1) acupuntura para reducir los síntomas de cáncer avanzado de colon y recto, 2) quimioterapia de combinación más radioterapia con o sin cartílago de tiburón en el tratamiento de pacientes que tienen cáncer de pulmón de células no pequeñas que no se puede extirpar con cirugía, 3) terapia de oxigenación hiperbárica en pacientes con laringectomía (personas que han tenido una operación para extirpar toda la laringe o una parte), 4) curación a distancia en el tratamiento de cáncer de cerebro, 5) quimioterapia comparada con terapia de enzimas de páncreas para el tratamiento de cáncer de páncreas, y 6) extracto de muérdago y quimioterapia para el tratamiento de tumores sólidos. Como está claro, no hay aún evidencia desde la ciencia, en relación con la efectividad de este tipo de tratamiento en la leucemia.
Con respecto a la creencia de que la quimioterapia es experimentación, no se hará al paciente oncológico ningún tratamiento cuyo protocolo no esté previamente probado y estudiado. En caso de quimiorresistencia, el hemato-oncólogo, decidirá la conducta terapéutica; ello no implica que lo que se ofrezca al paciente sea con objetivos experimentales. Los nuevos protocolos que requieran de estudio serán probados siempre con el consentimiento del paciente y bajo los más estrictos controles y principios éticos. Todos los medicamentos antineoplásicos disponibles en el mercado en la actualidad han sido estudiados con sumo cuidado y por lo tanto se tienen identificadas su farmacocinética y farmacodinámica, lo que por supuesto no sucede con las sustancias de origen homeopático o bioenergético de las cuales

\footnotetext{
* Chulo es sinónimo de buitre o golero en ciertas regiones de Colombia

** José Gregorio Hernández: médico nacido en Inotsú-Trujillo (Venezuela) en en I864. Tras hacer una carrera meritoria en Europa, regresa a Caracas en donde en 1919 es atropellado por un automóvil. Desde entonces, es venerado por sus virtudes como médico y por su vocación religiosa; tanto así que está en proceso de canonización y beatificación por parte de la iglesia católica. Se le reconocen virtudes de sanación; mencionándose que tiene el poder de curar toda clase de dolencias físicas y mentales y que lo hace a través de actos quirúrgicos para los cuales se desarrolla una serie de rituales en los que el espíritu de este médico opera al paciente.
} 
se desconoce desde su proceso de fabricación hasta sus mecanismos de acción, de excreción y su metabolismo.

Dado que una de las principales falsas creencias es que la leucemia es producto de estados anémicos previos, la práctica popular invita a tomar sangre de animales y a comer alimentos ricos en hierro. Otras personas deciden tomar suplemento de sulfato ferroso. Con respecto a estas prácticas, previamente se enunció que en efecto hay dos tipos de anemia que se asocian con el desarrollo de leucemias: la constitucional de Fanconi y la sideroblástica. Pero la leucemia en sí misma no tiene relación alguna con el déficit de hierro y el tratamiento estándar tampoco incluye su consumo. Por lo tanto, esta práctica puede causar consecuencias negativas en el paciente con leucemia por diversas razones, entre ellas porque el consumo de hierro se asocia a "molestia epigástrica, náusea, vómito, constipación o diarrea; estos efectos son más frecuentes si se usan dosis altas de hierro" (Gay Rodríguez, 1998) y pueden empeorar en forma significativa la percepción de bienestar subjetivo.

No obstante, el paciente con leucemia debería procurar tener una nutrición apropiada que favorezca su inmunocompetencia. Esto se traduce en que de ser posible, una alimentación balanceada proveerá al paciente de mejores condiciones para afrontar la quimioterapia. Incluye consumir alimentos ricos en nutrientes y entre ellos por supuesto estará el hierro, que está contenido en carnes y pescados. Sin embargo, no es pertinente comerlos crudos pues no reportan mayores beneficios y todo lo contrario, la manipulación inadecuada de estas carnes hace que su ingesta sin cocción pueda poner en riesgo la vida de los pacientes inmunosuprimidos, pues existe una mayor probabilidad de que microorganismos presentes en ellas ingresen al organismo causando graves consecuencias.

El consumo de sangre de chulo es una práctica muy común y que puede generar efectos muy nocivos en los pacientes. La creencia se asocia, según reporte de los pacientes en consulta, al hecho de que al ser este animal carroñero debe tener un sistema inmunológico muy fuerte y a través del consumo de su sangre y de las vísceras preparadas en caldo, se logra la inmunocompetencia del paciente.

En lo que respecta al consumo de plantas y extractos naturales para el tratamiento de la leucemia, los dos de mayor utilización son el anamú (Petiveria alliacea L) y el noni (Morinda citrifolia L).

El anamú es una planta perenne que crece en América del Sur que de acuerdo con Lemus Rodríguez et al. (2004), "posee comprobadas propiedades que estimulan la respuesta celular inmune, lo cual ha justificado elaborar las tabletas con tan importantes características y registrarlas como medicamento herbario inmunoestimulante, según los requisitos de la autoridad regulatoria cubana de medicamentos para uso humano" (p.57). Los usos etnomédicos de la planta contemplan fundamentalmente sus acciones como analgésico, antiinflamatorio (sobre todo en la artritis y gastritis) e hipoglucemiante y para el tratamiento del cáncer.

Al respecto, Jovicevic (1993), demostró el efecto inhibitorio de la Petiveria alliacea sobre la proliferación tumoral, en especial sobre líneas de células leucémicas, sin comprometer los tejidos normales. Así mismo, Marini et al. (1993), describieron la acción de los extractos de la planta para inducir la producción de linfocinas como la interleukina 2 y la 4, así como su capacidad para incrementar la actividad citotóxica de las células NK (natural killer). Otros estudios han demostrado el papel inmunomodulador de esta planta (Lad, 1997; Williams, 1997; Queiroz, 2000; y Rosner et al., 2001). No obstante, los trabajos no son concluyentes y por consiguiente no pueden hacerse afirmaciones con respecto a las mencionadas propiedades antitumorales de esta planta. Hay que anotar que si así lo fuera, es importante recordar que contiene coumarín y benzaldehído, fármacos utilizados como agentes antineoplásicos, pero la dosis antitumoral no se conoce y de hecho, estos medicamentos deben ser dosificados por el hemato-oncólogo si llegan a hacer parte de un protocolo de quimioterapia para leucemia. 
Sin embargo, se reconocen sus propiedades inmunoestimulantes y por ello, de ser considerada su administración, debe ser complementaria pues no existe evidencia alguna a nivel científico de propiedades curativas del cáncer. Incluso, según la investigación de Morón Rodríguez y Morón Pinedo (2004) el anamú no fue favorable cuando se asoció con paclitaxel, arabinósido de citosina o fármacos anticancerosos inmunosupresores como ciclofosfamida, metrotexato o 6-tioguanina, todos ellos agentes antineoplásicos.

Por su parte, el noni (Morinda citrifolia L.) "es una planta medicinal tradicional de la Polinesia, a la cual le atribuyen diversas propiedades; sin embargo, existen pocos trabajos publicados que validan científicamente su uso" (Morón Rodríguez y Morón Pinedo, 2004. p. 1).

Investigaciones recientes realizadas en modelos de matriz de coágulo de fibrina tridimensional con vena placentaria y de tumor de mama humanos para evaluar el desarrollo angiogénico vascular, indican que el jugo del fruto en ciertas concentraciones en el medio de cultivo, indujo degeneración vascular y apoptosis en los pozos con células capilares establecidas a los pocos días de haber sido aplicado (Morón Rodríguez y Morón Pinedo, 2004). En otro estudio realizado con ratas (Wang y $\mathrm{Su}, 2001$ ) se encontró que el jugo de esta fruta tuvo efecto antioxidante in vitro que fue comparable con el producido por la vitamina $\mathrm{C}$, polvo de semilla de uva y picnogenol en dosis equivalentes a las diarias recomendadas en los Estados Unidos, lo que explica que los autores sugirieran que podría contribuir a prevenir el cáncer.

Con respecto a lo anterior, debe anotarse que en la práctica clínica se evidencia una importante proporción de pacientes oncológicos que consume este tipo de extractos de plantas y de frutos. Lo cierto es que no se ha observado evidencia de que el mencionado consumo haya detenido el curso natural del cáncer, mientras que el abandono de la terapia oncológica y su reemplazo por el consumo de estos extractos sí permite observar un franco deterioro del paciente a nivel clínico. No se discute en este artículo el efecto que estas conductas tienen sobre el bienestar subjetivo del paciente.

En lo que se insiste en la terapia psicológica es que todo medicamento alopático u homeopático o de cualquier otra índole que el paciente decida tomar, debe ser consultado con su hemato-oncólogo, de manera que no genere interacciones medicamentosas o tóxicas indeseables. Además, se insiste en la importancia y pertinencia de no abandonar la quimioterapia. Se reconoce en la comunidad científica que el uso indiscriminado de este tipo de sustancias es un problema de salud pública a nivel mundial.

Como se ha demostrado antes, las creencias sobre la enfermedad se encuentran en relación con la etiología, tratamiento y pronóstico. Se analizó este último con las recaídas y ahora lo hacemos para indicar que el futuro del paciente dependerá en esencia del compromiso (responsabilidad y cumplimiento) con el tratamiento médico, de las diferencias individuales, pues cada organismo tiene una respuesta específica al tratamiento, y de las características y el estadio de la enfermedad al momento del diagnóstico.

Lo cierto es que conforme más falsas creencias tenga una persona en relación con el origen de la enfermedad, su tratamiento y pronóstico, será más complejo el ajuste y adaptación al proceso de enfermedad, es decir, más difícil será el afrontamiento de ésta.

Por lo tanto, el abordaje terapéutico de las creencias debe estar a cargo de los profesionales cuyo objeto de estudio es el comportamiento humano; es decir, de psicólogos, pues hay que considerar siempre que lo que subyace al abandono de una creencia es la pérdida de control sobre la realidad. Así que no se promoverá que un paciente abandone una de sus creencias, por falsa que esta sea, si no dispone de una idea mejor o de otra creencia que le permita un afrontamiento más adaptativo de la enfermedad. Así, la reestructuración se consolida como una técnica de abordaje psicoterapéutico de elección. No obstante, se describe a continuación otra estrategia 
para combatir las creencias falsas y generar un mejor afrontamiento de la enfermedad.

En primer lugar, el psicólogo entrenará al paciente para que reconozca la posibilidad de que existen falsas creencias y así pueda identificar las propias en relación con el origen de la enfermedad, su tratamiento y pronóstico. En segundo lugar, se le entrenará para que obtenga información aclarando que provendrá de fuentes válidas y confiables, en lo posible será científica o proporcionada por expertos en el tema, comprensible y suficiente para que se sienta ilustrado. Se motiva al paciente para que solicite tanta como requiera para satisfacer sus inquietudes. En tercer lugar, se prepara al paciente para que pueda elaborar todo el conocimiento que ahora dispone. Esto implica que esté en la capacidad de interrogarse si comprendió la información, indagar hasta comprenderla solicitando tantas explicaciones y soportes como le sea necesario, empezar a creer en lo que ha aprendido y comprendido, y decidirse a reemplazar, o en su defecto a reestructurar, sus falsas creencias. En cuarto lugar, el paciente recibirá entrenamiento para tomar decisiones. Este es un proceso complejo que consta de varias fases, cuya descripción no es objeto de este estudio. Sin embargo, se resalta que para lograr este proceso de toma de decisiones que se espera funcional, el psicólogo entrenará también al paciente para que desarrolle las habilidades pertinentes para contemplar diferentes alternativas para un problema que ya ha definido previamente, someterlas a juicio a través del reconocimiento de las ventajas y desventajas y costos y beneficios identificados; con ello, el paciente habrá de analizar las consecuencias que a nivel familiar, social y especialmente personal, puede traerle una decisión a corto, mediano y largo plazo. Esto implica que el paciente estará en capacidad de resolver el problema asociado con una falsa creencia, maximizar los beneficios potenciales de ello, minimizar los riesgos y elegir la solución que más ventajas le genere.

El efecto observado a partir de la experiencia clínica con el paciente oncológico mediante el procedimiento descrito, ha sido positivo. Se trata de una conclusión cualitativa pues en definitiva no existe un estudio que permita afirmar a cabalidad su efecto sobre los pacientes. No obstante, el trabajo sobre la creencia es un reto para el psicoterapeuta y está claro que en el campo de acción del psicólogo de la salud, no hay lugar a la realización de terapias muy prolongadas. Por consiguiente, la identificación precoz de creencias asociadas con la etiología y tratamiento de la leucemia, puede constituir un factor de éxito para aumentar la probabilidad de ajuste y adaptación al proceso de enfermedad. Esto implica también una mayor probabilidad de generar o fortalecer estrategias de afrontamiento activo dirigidas a la solución del problema y a la participación proactiva del paciente en su enfermedad, generando así mayor percepción de bienestar.

Para terminar, no debe ignorarse la convicción que la persona tiene de que la realización de determinada conducta le proporcionará tanto en lo teórico (agrado) como en lo práctico (utilidad), consecuencias favorables. Esto permite explicar qué lleva a personas en apariencia similares (en lo familiar, escolar, económico, cultural, social o por grupos de edad, por ejemplo) a comportarse de maneras distintas frente a los acontecimientos.

\section{Lecturas recomendadas}

- Abbas Z, Malik I, Khan A. Sequential induction of aplastic anemia and acute leukemia by chloramphenicol. J Pak Med Assoc. 1993 Mar;43(3):58-9

- Asociación entre vivir cerca de gasolineras y la leucemia en niños. Rev Panam Salud Publica 2004 Sep;16(3):209-10

- Atra A, Gerrard M, Hobson R, Imeson JD, Ashley S, Pinkerton CR. Improved cure rate in children with B-cell acute lymphoblastic leukaemia (B-ALL) and stage IV B-cell nonHodgkin's lymphoma (B-NHL)--results of the UKCCSG 9003 protocol. Br J Cancer. 1998 Jun;77(12):2281-5.

- Auvinen A, Hakama M, Arvela H, Hakulinen T, Rahola T, Suomela M, Soderman B, Rytomaa T. Fallout from Chernobyl and incidence of childhood leukaemia in Finland, 1976-92. BMJ. 1994 Jul 16;309(6948):151-4.

- Barros Kanzaki LI. Hypothetical HTLV-I induction by ionizing radiation. Med Hypotheses. 2006;67(1):177-82. Epub 2006 Mar 15

- Breckow J. [Evaluation of cancer risk by ionizing radiation after dosimetry revision of Hiroshima and Nagasaki]. Med Klin (Munich). 1991 Feb 15;86(2):92-8 
- Bosch JA, Berntson GG, Cacioppo JT, Marucha PT. Differential mobilization of functionally distinct natural killer subsets during acute psychologic stress. Psychosom Med. 2005 May-Jun;67(3):366-75.

- Bower JE, Ganz PA, Aziz N. Altered cortisol response to psychologic stress in breast cancer survivors with persistent fatigue. Psychosom Med. 2005 Mar-Apr;67(2):277-80

- Borducchi DM, Oliveira JS, Bordin JO, Kerbauy J. HTLV-I infection among relatives of patients with adult T-cell leukemia/lymphoma in Brazil: analysis of infection transmission. Leuk Lymphoma. 1998 Oct;31(3-4):411-6.

- Capuron L, Gumnick JF, Miller AH. Fundamentals of Psychoneuroimmunology. Psychosom Med. 2002 SepOct;64(5):848-9.

- Carlson LE, Speca M, Patel KD, Goodey E. Mindfulnessbased stress reduction in relation to quality of life, mood, symptoms of stress, and immune parameters in breast and prostate cancer outpatients. Psychosom Med. 2003 JulAug;65(4):571-81

- Derias EM, Stefanis P, Drakeley A, Gazvani R, Lewis-Jones DI. Growing concern over the safety of using mobile phones and male fertility. Arch Androl. 2006 Jan-Feb;52(1):9-14

- Fernandez-Ranana JM. Terapia en Oncohematologia. Barcelona: Harcourt Brace; 1998.

- Fette, A. A Pediatric Civil War Burn Victim: The Patient's and Caregivers' Stress and Coping. Plast Surg Nurs. 2005 April/June;25(2):66-71

- Fishbein M, Ajzen I, Belief, attitude, intention and behavior: an introduction to theory and research. USA: Addison Wesley; 1975.

- Frey AH. Hold the (cell) phone... Science. 2002 Jan 18:295(5554):440-1

- Gluzman D, Imamura N, Sklyarenko L, Nadgornaya V, Zavelevich M, Machilo V. Patterns of hematological malignancies in Chernobyl clean-up workers (1996-2005). Exp Oncol. 2006 Mar;28(1):60-3

- Gozdasoglu S, Cavdar AO, Arcasoy A, Babacan E, Sanal O. Fanconi's aplastic anemia, analysis of 18 cases. Acta Haematol. 1980;64(3):131-5

- Gutiérrez JD. La teoría de las representaciones sociales y sus implicaciones metodológicas en el ámbito psicosocial. Psiquiatr pública. 1998; 10(4):211-9

- Hardell L, Mild KH, Carlberg M, Hallquist A. Cellular and cordless telephone use and the association with brain tumors in different age groups. Arch Environ Health. 2004 Mar;59(3):132-7

- Hernández P. Leucemia linfoide crónica. Aspectos clínicos y biológicos. Rev Cubana Hematol Inmunol Hemoter. 1999;15(1):7-20

- Holland, JC. American Cancer Society Award lecture. Psychological care of patients: psycho-oncology's contribution. J Clin Oncol. 2003 Dec 1;21(23 Suppl):253s$265 \mathrm{~s}$.

- Holmberg M. Is the primary event in radiation-induced chronic myelogenous leukemia the induction of the $t(9 ; 22)$ translocation? Leuk Res. 1992;16(4):333-6.
- Jimenez JJ, Jimenez JG, Daghistani D, Yunis AA. Interaction of chloramphenicol and metabolites with colony stimulating factors: possible role in chloramphenicol-induced bone marrow injury.Am J Med Sci. 1990 Dec;300(6):350-3

- Jovicevic, L. In vitro antiproliferative activity of Petiveria alliacea on several tumor cell lines. Pharmacol Res 1993; 27(1):105-6.

- Kiecolt-Glaser JK, McGuire L, Robles TF, Glaser R. Psychoneuroimmunology and psychosomatic medicine: back to the future. Psychosom Med. 2002 Jan-Feb;64(1):1528

- Kiecolt-Glaser JK, Glaser R. Psychoneuroimmunology and health consequences: data and shared mechanisms. Psychosom Med. 1995 May-Jun;57(3):269-74

- Kiecolt-Glaser JK, Glaser R. Psychoneuroimmunology and immunotoxicology: implications for carcinogenesis. Psychosom Med. 1999 May-Jun;61(3):271-2

- Kishi Y, Kami M, Oki Y, Hamaki T, Kanda Y, Machida U, Miyakoshi S, Ueyama J, Morinaga S, Muto Y. Successful bone marrow transplantation for adult T-cell leukemia from a donor with oligoclonal proliferation of T-cells infected with human T-cell lymphotropic virus. Leuk Lymphoma. 2001 Aug;42(4):819-22.

- Khosravi Shahi P. [Plasma cell leukaemia: a rare variant of multiple myeloma. A case report] An Med Interna. 2005 Nov;22(11):532-4.

- Lad W. Inmunological activity of Petiveria alliacea. Phytother Res 1997;11:251-3.

- Lemus Z, García ME, Batista A, De la Guardia O, Castillo. La tableta de anamú: un medicamento herbario inmunoestimulante . Medisan. 2004;8(3):57-64.

- León M. Representaciones sociales: actitudes, creencias, comunicación y creencia social. En: Morales J., Páez D, Kornblit A, Asun D, editores. Psicología Social. Argentina: Prentice Hall; 2002.

- Lusk B, Lash AA. The stress response, psychoneuroimmunology, and stress among ICU patients. Dimens Crit Care Nurs. 2005 Jan-Feb;24(1):25-31.

- Magalhaes SM, Duarte FB, Ribeiro SC, Borovik CL, Lorand-Metze I. Sideroblastic anemia following treatment of chronic myeloid leukemia with busulfan. Leukemia. 2000 Jan;14(1):214-5.

- McNally RJ, Parker L. Environmental factors and childhood acute leukemias and lymphomas. Leuk Lymphoma. 2006 Apr;47(4):583-98.

- Milanés MT, Losada R, Hernández P, et al. Aspectos clínicos y epidemiológicos de la leucemia mieloide aguda en el anciano. Rev Cubana Hematol Inmunol Hemoter. 2002 Ene-Abr 18(1):0-0.

- Montserrat E. Leucemia linfática crónica: clínica, pronóstico y terapia. En: López Borrasca. Enciclopedia iberoamericana de Hematología. Salamanca: Universidad de Salamanca; 1992. p. 310-22.

- Morón FJ, Morón D. Mito y realidad de Morinda citrifolia L. (noni). Rev Cubana Plant Med. 2004;9(3). 
- Moulder JE, Foster KR, Erdreich LS, McNamee JP. Mobile phones, mobile phone base stations and cancer: a review. Int J Radiat Biol. 2005 Mar;81(3):189-203.

- Muirhead CR. Studies on the Hiroshima and Nagasaki survivors, and their use in estimating radiation risks. Radiat Prot Dosimetry. 2003;104(4):331-5. Review.

- Myers D. Psicología Social. México: McGraw-Hill; 1996.

- La medicina complementaria y alternativa en el tratamiento del cáncer [página de Internet] Mittwoch: Nacional Cancer Institute; 2002 [actualizado 06 Nov 2002; citado 12 Sep 2006] Disponible en: http://www.meb.uni-bonn.de/cancernet/spanish/600914.html.

- Newcom SR. Case 22-2005: intravenous immune globulin in chronic lymphocytic leukemia. N Engl J Med. 2005 Oct 27;353(17):1862-3; author reply 1862-3.

- Nunes MV, Tajara EH. [Delayed effects of organochlorine pesticides in man] Rev Saude Publica. 1998 Aug;32(4):37282. Portugues.

- Padua RA, West RR. Oncogene mutation and prognosis in the myelodysplastic syndromes. Br J Haematol. 2000 Dec;111(3):873-4.

- Pereira DB, Antoni MH, Danielson A, Simon T, EfantisPotter J, Carver CS, Duran RE, Ironson G, Klimas N, O'Sullivan MJ. Life stress and cervical squamous intraepithelial lesions in women with human papillomavirus and human immunodeficiency virus. Psychosom Med. 2003 May-Jun;65(3):427-34.

- Preston DL, Pierce DA, Shimizu Y, Cullings HM, Fujita S, Funamoto S, Kodama K. Effect of recent changes in atomic bomb survivor dosimetry on cancer mortality risk estimates. Radiat Res. 2004 Oct;162(4):377-89.

- Queiroz ML, Quadros MR, Santos LM. Cytokine profile and natural killer cell activity in Listeria monocytogenes infected mice treated orally with Petiveria alliacea extract. Immunopharmacol Immunotoxicol. 2000 Aug;22(3):50118.

- Rahu M, Rahu K, Auvinen A, Tekkel M, Stengrevics A, Hakulinen T, Boice JD Jr, Inskip PD. Cancer risk among Chernobyl cleanup workers in Estonia and Latvia, 19861998. Int J Cancer. 2006 Jul 1;119(1):162-8.

- Raison CL, Miller AH. Psychoneuroimmunology: stress, mental disorders and health . Psychosom Med. 2002 SepOct:64(5):847-8.
- Schwarz J, Pytlik R, Doubek M, Brychtova Y, Dulicek P, Campr V, Kren L, Penka M. Analysis of risk factors: the rationale of the guidelines of the Czech Hematological Society for diagnosis and treatment of chronic myeloproliferative disorders with thrombocythemia. Semin Thromb Hemost. 2006 Apr;32(3):231-45.

- Tacon AM. Attachment experiences in women with breast cancer. Fam Community Health. 2003 Apr-Jun;26(2):14756.

- Torres D, Capote T. Agroquímicos un problema ambiental global: uso del análisis químico como herramienta para el monitoreo ambiental. Ecosistemas. Revista científica y técnica de ecología y medio ambiente. [seriada en Internet] 2004 [citado 2006 Sep 12]. Disponible en: http://www.revistaecosistemas.net/index_frame. asp?pagina $=\mathrm{http} \% 3 \mathrm{~A} / \mathrm{www}$.revistaecosistemas.net/articulo. asp $\% 3$ FId $\% 3$ D50\%26Id_Categoria\%3D1\%26tipo \%3Dpo rtada.

- Martínez, JL. Anemia del prematuro. Estrategias terapéuticas. Revista Médica de Clínica Las Condes [seriada en Internet] 1999 Abr [citado 2006 Sep 12];10(1):[aprox. 6 p.]. Disponible en http://www.clc.cl/area_academica/ Revista\%20Medica\%20Abril\%201999/articulo_004.htm

- Tosevski DL, Milovancevic MP. Stressful life events and physical health. Curr Opin Psychiatry. 2006 Mar;19(2):1849.

- Uhlig T, Kallus KW. The brain: a psychoneuroimmunological approach. Curr Opin Anaesthesiol. 2005 Apr;18(2):14750.

- Valle L, Fernandez V, Perez-Pons C, Sanchez FG, Benitez $\mathrm{J}$, Urioste M. Generation of the BCR/ABL fusion gene in a Philadelphia chromosome-negative chronic myeloid leukaemia: insertion of $5.6 \mathrm{Mb}$ of $9 \mathrm{q} 34$ into the BCR region of chromosome 22. Hematol Oncol. 2006 Jun;24(2):86-8.

- Vergara-Rodriguez P. Human psychoneuroimmunology. Shock. 2006 Mar;25(3):317-8.

- Wang MY, Su C. Cancer preventive effect of Morinda citrifolia (Noni). Ann N Y Acad Sci. 2001 Dec;952:161-8.

- Whitfield CL. Psychoneuroimmunology: stress, mental disorders and health. J Nerv Ment Dis. 2002 Feb;190(2):12930. 